\title{
Phase II study of olaparib in patients with refractory Ewing sarcoma following failure of standard chemotherapy
}

Edwin Choy ${ }^{1,4,7^{*}}$, James E Butrynski ${ }^{2}$, David C Harmon ${ }^{1,4}$, Jeffrey A Morgan², Suzanne George ${ }^{2}$, Andrew J Wagner ${ }^{2}$, David D'Adamo ${ }^{2}$, Gregory M Cote ${ }^{1,4}$, Yael Flamand ${ }^{3}$, Cyril H Benes ${ }^{4}$, Daniel A Haber ${ }^{1,4}$, Jose M Baselga $a^{1,4,6}$ and George D Demetri ${ }^{2,5}$

\begin{abstract}
Background: Preclinical studies have documented antitumor activity of PARP inhibition both in vitro and in vivo, against Ewing sarcoma cells. This study aimed to translate that observation into a clinical trial to assess the efficacy and tolerability of olaparib, a PARP inhibitor, in patients with advanced Ewing sarcoma (EWS) progressing after prior chemotherapy.

Methods: In this nonrandomized phase II trial, adult participants with radiographically measureable metastatic EWS received olaparib tablets, $400 \mathrm{mg}$ orally twice daily, until disease progression or drug intolerance. Tumor measurements were determined by CT or MRI at 6 and 12 weeks after starting olaparib administration, and then every 8 weeks thereafter. Tumor response determinations were made according to RECIST 1.1, and adverse event determinations were made according to CTCAE, version 4.0. A total of 22 participants were planned to be enrolled using a conventional 2-step phase II study design. If no objective responses were observed after 12 participants had been followed for at least 3 months, further accrual would be stopped.
\end{abstract}

Results: 12 participants were enrolled, and all were evaluable. There were no objective responses (PR/CR), 4 SD (duration 10.9, 11.4, 11.9, and $17.9 \mathrm{wks}$ ), and 8 PD as best response. Of the SD, 2 had minor responses (-9\% and $-11.7 \%$ by RECIST 1.1). The median time to disease progression was 5.7 weeks. Further enrollment was therefore discontinued. No significant or unexpected toxicities were observed with olaparib, with only a single case each of grade 3 anemia and grade 3 thrombocytopenia observed.

Conclusions: This study is the first report of a prospective phase II trial to evaluate the safety and efficacy of a PARP inhibitor in patients with advanced Ewing sarcoma after failure of standard chemotherapy. Olaparib administration was safe and well tolerated when administered to this small heavily pre-treated cohort at the $400 \mathrm{mg}$ BID dose, although the median duration of dosing was for only 5.7 weeks. No significant responses or durable disease control was seen, and the short average interval to disease progression underscores the aggressiveness of this disease. Other studies to combine cytotoxic chemotherapy with PARP inhibition in EWS are actively ongoing.

Trial registration: ClinicalTrials.gov Identifier: NCT01583543

\footnotetext{
* Correspondence: echoy@mgh.harvard.edu

'Division of Hematology Oncology, Massachusetts General Hospital, Boston, MA, USA

${ }^{4}$ Massachusetts General Hospital Cancer Center, Boston, MA, USA

Full list of author information is available at the end of the article
} 


\section{Background}

Ewing sarcoma is a highly malignant tumor of either bone or soft tissue that occurs most frequently in the adolescent and young adult years [1]. The cell of origin of Ewing sarcoma remains poorly defined, however a neuroectodermal origin is suspected. Although a rare disease, it is the 2nd most common primary bone tumor of childhood. With the advent of adjuvant chemotherapy, the prognosis of localized Ewing sarcoma has improved from less than $20 \%$ to currently greater than $70 \%$ survival in 5-years. However, patients with recurrent Ewing sarcoma have poor prognosis [2-5]. Few patients with recurrences become long-term survivors, and they are usually limited to those with local recurrence and a long initial remission [4,6,7]. Second line and subsequent chemotherapy has had limited success and is associated with significant toxicity. There is no single accepted standard of care that is highly effective for these patients, and complete responses are rare. Five-year event-free and overall survival following recurrence is less than $15 \%[3,5,8]$. The prognosis is even worse for patients who relapse within two years after diagnosis and patients who have distant recurrences that are not treatable with radical surgery. In a single institution longitudinal experience over 20 years, 215 patients of an initial cohort of 402 patients $(53.5 \%)$ relapsed. Of these relapsed patients, 200 (93\%) died with a mean survival of 13.1 months and no patient with extrapulmonary metastases survived [8].

Garnett et al. observed that Ewing sarcoma cell lines were over 100-fold more sensitive to PARP inhibition with olaparib (AZD2281, KU-0059436), a potent Polyadenosine 5'diphophoribose (poly ADP ribose) polymerase (PARP) inhibitor, than were control cell lines, and treatment with Olaparib selectively induced apoptosis [9]. This level of sensitivity was comparable to that observed in BRCA2 deficient cells. Olaparib has been shown to inhibit selected tumor cell lines in vitro and in xenograft and primary explant models as well as in genetic BRCA knock-out models, either as a stand- alone treatment or in combination with established chemotherapies [10-12]. Brenner et al. additionally showed that PARP inhibition by olaparib potentiated DNA damage induced by expression of EWS-FLI1 or EWS-ERG fusion genes, thereby inhibiting growth of tumor subcutaneously implanted into SCID mice [13]. Based on these results, we performed a single arm open labeled clinical trial, constructed along a conventional Simon 2-step phase II study design [14], to evaluate the safety and clinical activity of olaparib in adult patients with advanced Ewing sarcoma following failure of conventional chemotherapy. (ClinicalTrials. gov Identifier: NCT01583543).

\section{Methods}

This study is a single arm, open label, phase II study to investigate the clinical efficacy and safety of olaparib in patients with metastatic and/or recurrent Ewing sarcoma. Pathologic diagnosis of Ewing sarcoma had to be confirmed by pathologic review at one of the participating institutions, but molecular testing for an EWS translocation was not required for eligibility.

A single consortium, the Dana-Farber/Harvard Cancer Center completed the study through two active sites

\section{Table 1 Baseline characteristics}

\begin{tabular}{|c|c|}
\hline \multicolumn{2}{|c|}{ Age (in years, at date of registration) } \\
\hline Mean & 30.5 \\
\hline Std & 15.38 \\
\hline Min & 18 \\
\hline Max & 70 \\
\hline \multicolumn{2}{|l|}{ Gender } \\
\hline Male & $N=10,83 \%$ \\
\hline Female & $N=2,17 \%$ \\
\hline \multicolumn{2}{|l|}{ Institution } \\
\hline $\mathrm{DFCl}$ & $\mathrm{N}=4,33.3 \%$ \\
\hline MGH & $N=8,66.7 \%$ \\
\hline \multicolumn{2}{|l|}{ Performance status } \\
\hline 0 & $N=6,50 \%$ \\
\hline 1 & $N=6,50 \%$ \\
\hline \multicolumn{2}{|l|}{ Prior surgery } \\
\hline Yes & $N=9,75 \%$ \\
\hline No & $N=3,25 \%$ \\
\hline \multicolumn{2}{|l|}{ Prior radiation } \\
\hline Yes & $N=9,75 \%$ \\
\hline No & $N=3,25 \%$ \\
\hline \multicolumn{2}{|c|}{ Number of prior radiation treatments } \\
\hline N (patients) & 9 \\
\hline Mean (\#prior treatments) & 1.7 \\
\hline Std & 1.1 \\
\hline Median & 1.0 \\
\hline Min & 1 \\
\hline Max & 4 \\
\hline \multicolumn{2}{|l|}{ Prior Chemotherapy } \\
\hline Yes & $\mathrm{N}=12,100 \%$ \\
\hline \multicolumn{2}{|c|}{ Number of prior chemotherapy treatments } \\
\hline N (patients) & 12 \\
\hline Mean (\#prior treatments) & 6.5 \\
\hline Std & 6.4 \\
\hline Median & 5 \\
\hline Min & 1 \\
\hline Max & 20 \\
\hline
\end{tabular}


(Dana-Farber Cancer Institute and Massachusetts General Hospital).

The study was designed to distinguish a favorable true response rate of $24 \%$ from a null rate of $5 \%$ using a conventional 2 -step phase II study design model [14]. The first group of 12 subjects was to be enrolled. If no patient experienced response after all 12 subjects had been

Table 2 Toxicity maximum grade per patient - attributable to treatment

\begin{tabular}{|c|c|c|c|c|}
\hline Toxicity & Description & Grade & & \\
\hline & & 1 & 2 & 3 \\
\hline BL101 & Anemia & 0 & 0 & 2 \\
\hline BL999 & $\begin{array}{l}\text { Blood and lymphatic system } \\
\text { disorders - Other }\end{array}$ & 1 & 0 & 0 \\
\hline CN108 & Fatigue & 3 & 0 & 0 \\
\hline CN109 & Fever & 2 & 0 & 0 \\
\hline CN121 & Non-cardiac chest pain & 1 & 0 & 0 \\
\hline Gl121 & Constipation & 1 & 0 & 0 \\
\hline Gl123 & Diarrhea & 1 & 0 & 0 \\
\hline Gl124 & Dry mouth & 2 & 0 & 0 \\
\hline Gl179 & Nausea & 2 & 1 & 0 \\
\hline Gl210 & Stomach pain & 1 & 0 & 0 \\
\hline Gl216 & Vomiting & 3 & 0 & 0 \\
\hline G1999 & $\begin{array}{l}\text { Gastrointestinal } \\
\text { disorders - Other }\end{array}$ & 1 & 0 & 0 \\
\hline IN171 & Urinary tract infection & 1 & 0 & 0 \\
\hline IV127 & Lymphocyte count decreased & 0 & 0 & 1 \\
\hline IV131 & Platelet count decreased & 0 & 1 & 1 \\
\hline ME999 & $\begin{array}{l}\text { Metabolism and nutrition } \\
\text { disorders - Other }\end{array}$ & 2 & 0 & 0 \\
\hline MU112 & Generalized muscle weakness & 1 & 0 & 0 \\
\hline MU999 & $\begin{array}{l}\text { Musculoskeletal and } \\
\text { connective tissue } \\
\text { disorder - Other }\end{array}$ & 1 & 0 & 0 \\
\hline NE118 & Dysgeusia & 1 & 0 & 0 \\
\hline NE126 & Headache & 1 & 0 & 0 \\
\hline PU113 & Cough & 1 & 0 & 0 \\
\hline PU999 & $\begin{array}{l}\text { Respiratory, thoracic and } \\
\text { mediastinal disorders - Other }\end{array}$ & 1 & 0 & 0 \\
\hline VA102 & Flushing & 1 & 0 & 0 \\
\hline VA104 & Hot flashes & 1 & 0 & 0 \\
\hline TOTAL & & 29 & 2 & 4 \\
\hline \multicolumn{5}{|c|}{ Worst grade per patient across all types } \\
\hline & & & Count & Percentage \\
\hline \multicolumn{3}{|c|}{ Mild - grade 1 as worst degree } & 5 & $45.45 \%$ \\
\hline \multicolumn{3}{|c|}{ Moderate - grade 2 as worst degree } & 1 & $9.09 \%$ \\
\hline \multicolumn{3}{|c|}{ Severe - grade 3 as worst degree } & 4 & $36.36 \%$ \\
\hline \multicolumn{3}{|c|}{ Life threatening } & 1 & $9.09 \%$ \\
\hline
\end{tabular}

followed for at least 3 months, further accrual would be stopped and the study drug would be declared as ineffective. If 1 or more patients experienced response, an additional 10 patients would be enrolled to a total study population of $\mathrm{N}=22$. If 3 or more patients among 22 eligible, treated patients experience response, the drug would be considered effective and worthy of further study. Study-wide response rates were to be estimated after all subjects had been followed for at least 6 months. This statistical model assumes a null versus alternative response rate as $5 \%$ versus $24 \%$, with a $9 \%$ type I error and $91 \%$ power. This design had a $54 \%$ probability of stopping early if the drug was ineffective.

Olaparib was administered orally at $400 \mathrm{mg}$ (tablet formulation) twice daily. Participants were to take Olaparib twice daily, without break, and were supplied a sufficient quantity on Day 1 of the study to last until the second study visit (Day 43). After Day 1 of the study additional visits occurred after 6 weeks, 12 weeks, and then every 8 weeks thereafter. Subjects were instructed not to make up for vomited doses, and to record missed or vomited doses on the Patient Diary Card. Safety and tolerability were monitored continuously throughout study participation.

Tumor assessments using CT scans of the chest, abdomen, and pelvis were done at baseline and then repeated after 6 weeks, 12 weeks, and then every 8 weeks thereafter to assess disease status.

\section{Table 3 Summary details}

\begin{tabular}{|c|c|}
\hline \multicolumn{2}{|l|}{ Reason treatment ended: } \\
\hline Progressive disease & $\mathrm{N}=12,100 \%$ \\
\hline \multicolumn{2}{|l|}{ Best response: } \\
\hline Stable disease & $\mathrm{N}=4,33.33 \%$ \\
\hline Progressive disease & $N=8,66.67 \%$ \\
\hline \multicolumn{2}{|l|}{ Number of treatment weeks completed } \\
\hline N & 12 \\
\hline Mean & 7.9 \\
\hline Std & 4.72 \\
\hline Min & 4 \\
\hline Max & 20 \\
\hline \multicolumn{2}{|l|}{ Duration of stable disease (weeks) } \\
\hline $\mathrm{N}$ & 4 \\
\hline Mean & 13.03 \\
\hline Std & 3.28 \\
\hline Min & 10.9 \\
\hline Max & 17.9 \\
\hline \multicolumn{2}{|c|}{ Progression-free survival: median of 5.7 weeks. } \\
\hline \multicolumn{2}{|l|}{ Survival status } \\
\hline Alive & $\mathrm{N}=1,8.33 \%$ \\
\hline Dead & $N=11,91.67 \%$ \\
\hline
\end{tabular}




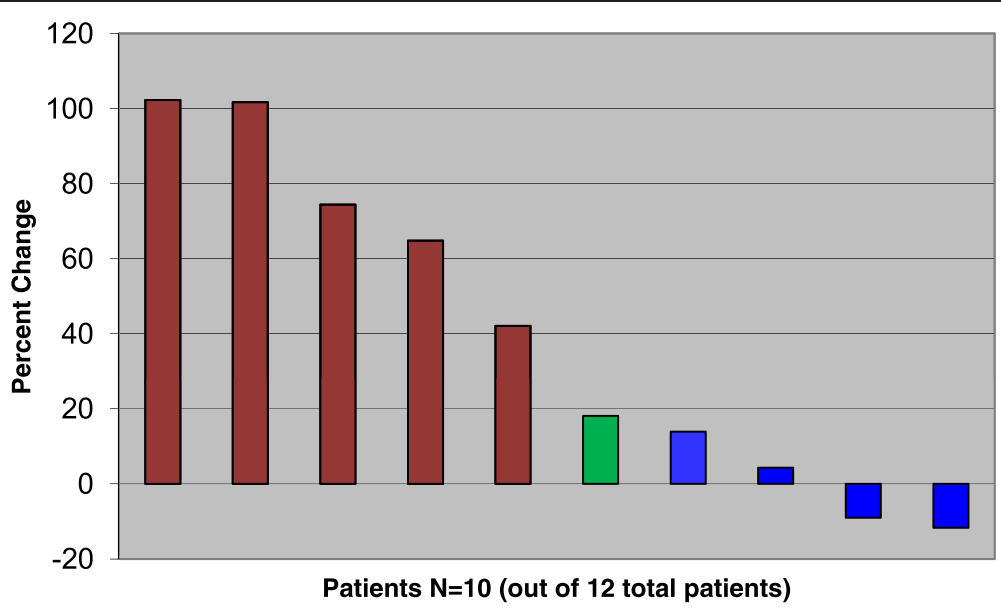

Figure 1 Percent change from baseline in sum of longest diameter of target lesions. Red: Progressive Disease, Green: Progressive Disease due to development of new metastatic lesions, Blue: Stable Disease. Two patients did not receive post-treatment imaging due to rapid clinical progression of disease.

In the absence of treatment delays due to adverse events, study drug administration was to be continued until one of the following criteria applied: Disease progression by RECIST 1.1, intercurrent illness that prevented further administration of treatment, unacceptable adverse events, decision of participant to withdraw from study, general or specific changes in the participant's condition that rendered the participant unacceptable for further treatment in the opinion of the treating investigator, or bone marrow findings consistent with myelodysplastic syndrome/acute myeloid leukemia.

The study was approved by the Dana-Farber/Harvard Cancer Center Institutional Review Board, and all patients signed informed consent prior to study registration. This study was opened to accrual on May 25, 2012, and closed to accrual on February 25, 2013. All patients were to be followed for at least 30 days after removal from study or until death. Participants who were removed from study for unacceptable adverse events were to be followed until resolution or stabilization of the adverse event.

\section{Results}

Table 1 displays patient demographics and other characteristics at baseline. The median age was 25.5 years (range 18 to 70 years). $100 \%$ of subjects were white, and $10(83 \%)$ were male, while $2(17 \%)$ were female. 9 subjects (75\%) had prior surgical treatment, and 9 subjects (75\%) had prior radiation treatment. The median number of prior radiation treatments was 1 and the median number of prior chemotherapy treatments was 5 .

Table 2 displays a summary of treatment-related adverse events (counts by worst grade per patient, and worst grade per patient across all toxicities). No deaths were experienced on this study. There was one occurrence of a Grade 4 event (patient was hospitalized with a pneumonia), and 6 occurrences of Grade 3 events (pain, anemia, lymphocyte count decreased and platelet count decreased) across all toxicities. The grade 4 event and 2 of the grade 3 events (pain) were not attributed to the study drugs or procedures by the study investigators. No patients were taken off study due to an unacceptable

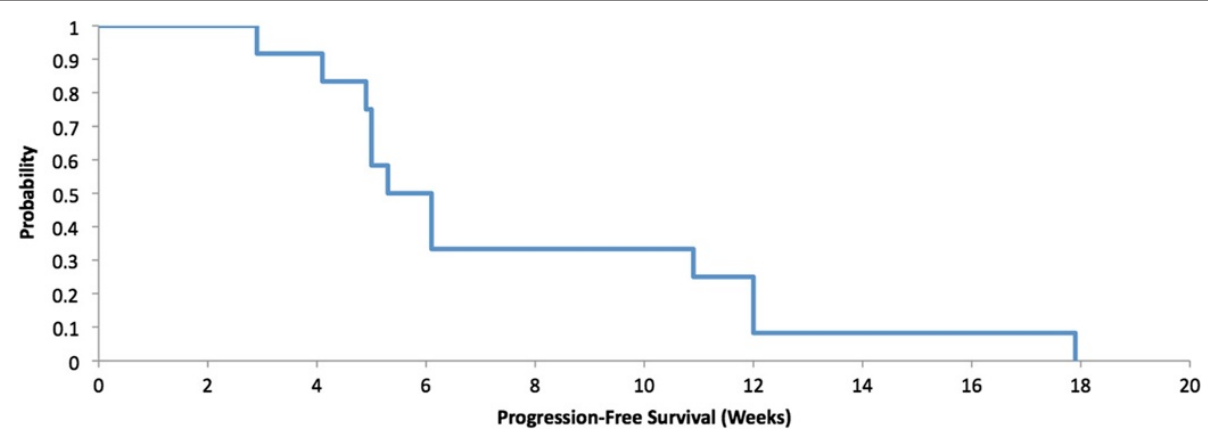

Figure 2 Progression free survival. 
adverse event. One patient did not experience any toxicity while on study treatment.

Table 3: Sixty-seven percent of the study subjects $(\mathrm{N}=8)$ experienced Progressive Disease as their best response, and $33 \%$ of the study subjects $(\mathrm{N}=4)$ experienced Stable Disease (Figure 1). Response was measured using RECIST criteria version 1.1. Table 3 illustrates summary data and shows that the duration of stable disease had a median value of 11.6 weeks $(\mathrm{N}=4)$, with a minimum value of 10.9 weeks and a maximum value of 17.9 weeks. Progression free-survival had a median of 5.7 weeks (Figure 2). The $90 \%$ Confidence Interval for the response rate is $\{0-22 \%\}$.

\section{Discussion}

In summary, 12 participants with metastatic Ewing sarcoma were enrolled, and all were evaluable. Two cases of grade 3 anemia, one case of grade 3 thrombocytopenia, one case of grade 3 decreased lymphocyte count, and one case of grade 4 other respiratory, thoracic and mediastinal disorder were observed. Otherwise, no significant or unexpected toxicities were observed while participants were receiving olaparib. There were $0 \mathrm{PR} / \mathrm{CR}, 4 \mathrm{SD}$ (duration $10.9,11.4,11.9$, and $17.9 \mathrm{wks}$ ), and $8 \mathrm{PD}$ as best response. Of the participants who experienced SD, 2 had minor responses ( $-9.0 \%$ and $-11.7 \%$ by RECIST 1.1 , see Figure 1$)$. Further enrollment was therefore discontinued after interim analysis.

This lack of clinical efficacy is in direct contrast to preclinical modeling that supported in vitro sensitivity of Ewing sarcoma cell lines to olaparib [9]. One explanation for this discrepancy is that some cell lines may have been derived from patient tumor samples that were not yet chemoresistant, while all participants in this study had proven relapse or progression after administration of standard chemotherapeutic agents for Ewing sarcoma. Several other factors that could explain the disparity include failure to achieve in vitro levels of olaparib at the clinical dose, secondary genomic or epigenomic alterations that might have activated other drivers of tumor cell proliferation that would render the PARP pathway nonessential, or yet unidentified mediators of PARPrescue derived from tumor-environment interactions.

Preclinical work, however, does support potential activity of PARP inhibition when combined with alkylating agents. In fact, although Brenner et al. showed that in mice xenografts, Ewing sarcoma (RD-ES) cells treated with $100 \mathrm{mg} / \mathrm{kg}$ of olaparib twice a day still demonstrated tumor growth, albeit significantly slower than untreated controls [13], in that same report, olaparib combined with the alkylating agent, temozolomide, yielded exquisite and durable in vivo tumor response. Such trials combining PARP inhibition with chemotherapy are currently being conducted or under development at several sites across the U.S. and in Europe.

\section{Conclusion}

This study is the first report of a prospective phase II trial to evaluate the safety and efficacy of a PARP inhibitor in patients with Ewing sarcoma. Olaparib tablets were well tolerated when administered to this small cohort at the $400 \mathrm{mg}$ BID dose, although the median duration of dosing was for only 5.7 weeks. However, no significant responses were seen, and the short average interval to disease progression underscores the aggressiveness of this disease.

\section{Competing interests}

EC: Amgen, Bayer, NPS. JB: Novartis, Roche, Merck, Sanofi-Aventis, Verastem, Bayer, Chugai, Exelixis, Onyx, and Constellation. GD: Novartis, Pfizer, Sanofi-Aventis, Johnson \& Johnson, Merrimack Pharma, Foundation Medicine, Merck, Ariad, ZioPharm, Glaxo-Smith-Kline, Koltan Pharmaceuticals, Blueprint Medicines, G-1 Therapeutics, Champions Biotechnology, N-of-One. JB, DCH, JM, SG, AW, DD, GC, YR, CB, DAH: These authors declare that they have no competing interests.

\section{Authors' contributions}

EC wrote the protocol, implemented the clinical trial, and wrote the manuscript. JB, DCH, JM, SG, AW, DD, and GC participated in acquisition of data. YR participated in its design and helped to draft the manuscript. CB, $\mathrm{DAH}, \mathrm{JB}$, and GD made substantial contributions to conception and design of the study. GD also revised the manuscript critically for intellectual content. All authors read and approved the final draft of the manuscript for submission.

\section{Acknowledgements}

This study was supported by the Jennifer Hunter Yates Foundation, Ludwig Center at Dana-Farber / Harvard Cancer Center, the Brian Maclsaac Sarcoma Fund, and the National Cancer Institute of the National Institutes of Health under Award Number U54CA168512. The content is solely the responsibility of the authors and does not necessarily represent the official views of the National Institutes of Health.

\section{Author details}

'Division of Hematology Oncology, Massachusetts General Hospital, Boston, MA, USA. ${ }^{2}$ Medical Oncology, Dana Farber Cancer Institute, Boston, MA, USA. ${ }^{3}$ Department of Statistics, Dana Farber Cancer Institute, Boston, MA, USA. ${ }^{4}$ Massachusetts General Hospital Cancer Center, Boston, MA, USA. ${ }^{5}$ Ludwig Center at Dana-Farber/Harvard Cancer Center, Boston, MA, USA. ${ }^{6}$ Current address: Memorial Sloan Kettering Cancer Center, New York, NY, USA.

${ }^{7}$ Current address: 55 Fruit St., Boston, MA 02114, USA.

Received: 29 June 2014 Accepted: 2 October 2014

Published: 5 November 2014

\section{References}

1. Ross KA, Smyth NA, Murawski CD, Kennedy JG: The biology of ewing sarcoma. ISRN Oncol 2013, 2013:759725.

2. Bacci G, Ferrari S, Mercuri M, Longhi A, Giacomini S, Forni C, Bertoni F, Manfrini M, Barbieri E, Lari S, Donati D: Multimodal therapy for the treatment of nonmetastatic Ewing sarcoma of pelvis. J Pediatr Hematol Oncol 2003, 25:118-124.

3. MCTiernan AM, Cassoni AM, Driver D, Michelagnoli MP, Kilby AM, Whelan JS: Improving outcomes after relapse in Ewing's Sarcoma: analysis of 114 patients from a single institution. Sarcoma 2006, 2006:83548.

4. Rodriguez-Galindo C, Billups CA, Kun LE, Rao BN, Pratt CB, Merchant TE, Santana VM, Pappo AS: Survival after recurrence of Ewing tumors: the St Jude Children's Research Hospital experience, 1979-1999. Cancer 2002, 94:561-569. 
5. Shankar AG, Ashley S, Craft AW, Pinkerton CR: Outcome after relapse in an unselected cohort of children and adolescents with Ewing sarcoma. Med Pediatr Oncol 2003, 40:141-147.

6. Al-Faris N, Al Harbi T, Goia C, Pappo A, Doyle J, Gassas A: Does consolidation with autologous stem cell transplantation improve the outcome of children with metastatic or relapsed Ewing sarcoma? Pediatr Blood Cancer 2007, 49:190-195.

7. Barker LM, Pendergrass TW, Sanders JE, Hawkins DS: Survival after recurrence of Ewing's sarcoma family of tumors. J Clin Oncol 2005, 23:4354-4362.

8. Bacci G, Longhi A, Ferrari S, Mercuri M, Versari M, Bertoni F: Prognostic factors in non-metastatic Ewing's sarcoma tumor of bone: an analysis of 579 patients treated at a single institution with adjuvant or neoadjuvant chemotherapy between 1972 and 1998. Acta Oncol 2006, 45:469-475.

9. Garnett MJ, Edelman EJ, Heidorn SJ, Greenman CD, Dastur A, Lau KW, Greninger P, Thompson IR, Luo X, Soares J, Liu Q, lorio F, Surdez D, Chen L, Milano RJ, Bignell GR, Tam AT, Davies H, Stevenson JA, Barthorpe S, Lutz SR, Kogera F, Lawrence K, McLaren-Douglas A, Mitropoulos X, Mironenko T, Thi H, Richardson L, Zhou W, Jewitt F, et al: Systematic identification of genomic markers of drug sensitivity in cancer cells. Nature 2012, 483:570-575.

10. Hutchinson L: Targeted therapies: PARP inhibitor olaparib is safe and effective in patients with BRCA1 and BRCA2 mutations. Nat Rev Clin Oncol 2010, 7:549.

11. Khan OA, Gore M, Lorigan P, Stone J, Greystoke A, Burke W, Carmichael J, Watson AJ, McGown G, Thorncroft M, Margison GP, Califano R, Larkin J, Wellman S, Middleton MR: A phase I study of the safety and tolerability of olaparib (AZD2281, KU0059436) and dacarbazine in patients with advanced solid tumours. Br J Cancer 2011, 104:750-755.

12. Kortmann U, McAlpine JN, Xue H, Guan J, Ha G, Tully S, Shafait S, Lau A, Cranston AN, O'Connor MJ, Huntsman DG, Wang Y, Gilks CB: Tumor growth inhibition by olaparib in BRCA2 germline-mutated patient-derived ovarian cancer tissue xenografts. Clin Cancer Res 2011, 17:783-791.

13. Brenner JC, Feng FY, Han S, Patel S, Goyal SV, Bou-Maroun LM, Liu M, Lonigro R, Prensner JR, Tomlins SA, Chinnaiyan AM: PARP-1 inhibition as a targeted strategy to treat Ewing's sarcoma. Cancer Res 2012, 72:1608-1613.

14. Simon R: Optimal two-stage designs for phase II clinical trials. Control Clin Trials 1989, 10:1-10.

doi:10.1186/1471-2407-14-813

Cite this article as: Choy et al:: Phase II study of olaparib in patients with refractory Ewing sarcoma following failure of standard chemotherapy. BMC Cancer 2014 14:813.

\section{Submit your next manuscript to BioMed Central and take full advantage of:}

- Convenient online submission

- Thorough peer review

- No space constraints or color figure charges

- Immediate publication on acceptance

- Inclusion in PubMed, CAS, Scopus and Google Scholar

- Research which is freely available for redistribution 